
AB0497

EFFECTIVENESS, TOLERABILITY, AND SAFETY OF TOFACITINIB IN RHEUMATOID ARTHRITIS: A RETROSPECTIVE ANALYSIS OF REAL-WORLD DATA FROM THE ST. GALLEN AND AARAU COHORT

R. Mueller ${ }^{1}$, F. Popp ${ }^{1}$, F. Mattow ${ }^{1}$, M. Durmisi ${ }^{2}$, A. Souza ${ }^{3}$, H. Schulze-Koops ${ }^{4}$ P. Hasler ${ }^{2}$, J. von Kempis ${ }^{1}{ }^{1}$ Department of Rheumatology, Kantonsspital St. Gallen, St. Gallen; ${ }^{2}$ Division of Rheumatology, Kantonsspital Aarau, Aarau; ${ }^{3}$ Iterata AG, Gränichen, Switzerland; ${ }^{4}$ Rheumaeinheit, University of Munich, München, Germany

Background: Tofacitinib is an oral JAK inhibitor indicated for the treatment of RA Efficacy and safety of tofacitinib have been shown in several randomised clinical studies.

Objectives: The study presented here aimed to assess the clinical tolerability and effectiveness of tofacitinib among patients with RA in real life.

Methods: Consecutive patients between June 2013 and April 2017 with RA who fulfilled the American College of Rheumatology/EULAR 2010 criteria were analysed in a prospectively designed analysis of retrospective data. Patients were initiated on tofacitinib $5 \mathrm{mg}$ bid. The primary objective was to analyse safety of tofacitinib in a real life cohort. Safety was assessed by the reasons to stop tofacitinib during follow up and changes of liver enzymes, haemoglobin, and creatinine. The secondary outcome was to analyse the frequency of and time to achieve low disease activity (LDA) and remission as defined by DAS28.

Results: Overall, 144 patients were treated with tofacitinib. $84.9 \%$ of the patients were pre-exposed to at least one biological agent. The average DAS 28 at initiation of tofacitinib was $4.43 .50 .0 \%$ were rheumatoid factor and $49.0 \%$ ACPA positive. The mean follow up was 1.22 years (range $10 \mathrm{~d}-3.7 \mathrm{a}$ ) after initiation of tofacitinib treatment. 94 (64.4\%) patients remained on tofacitinib during follow up. The average time to stop tofacitinib was 190.0 days. Reasons to stop tofacitinib were: insufficient response $(n=23)$, gastrointestinal symptoms $(n=18)$, infection $(n=5)$, myalgia $(n=2)$, remission $(n=2)$, headache ${ }^{2}$ cough, blue toe syndrome, intolerance, heart burn, psoriasis, and increased liver enzymes (all $n=1$ ). Increased ALAT or ASAT >2 $\times$ ULN were detected in $3.3 \%$ and $4.4 \%$, respectively. These elevated transaminase levels were transient in $50 \%$ and $60 \%$ of the cases, respectively. Haemoglobin decrease of $>10 \%$ was detected in $15.1 \%$ of the patients and decreased lymphocytes $<500 / \mu \mathrm{l}$ in $3.4 \%$. An increase of creatinine $>20 \%$ was detected in $9.4 \%$.

$62.9 \%$ and $50.0 \%$ of the patients achieved LDA or remission after a median 260 and 616 days, respectively. These rates were significantly higher in patients naïve to biologic agents as compared to patients pre-exposed to biologics (LDA: naïve $100 \%$ after median $100 \mathrm{~d}$, pre-exposed $57.0 \%$ after $359 \mathrm{~d}$; remission: naïve $86.7 \%$ after $132 \mathrm{~d}$, pre-exposed $44.1 \%$ after 720 )

Conclusions: Tofacitinib is a safe and effective treatment option for patients with RA. Tofacitinib may induce high rates of LDA and remission in patients with active disease, even after use of one or more biologics, though the rate is significantly higher in patients naïve to biologics. Tofacitinib may be a valuable option in a treat to target approach. Our data justify an early use of tofacitinib in the therapeutic strategy.

Disclosure of Interest: None declared

DOI: 10.1136/annrheumdis-2018-eular.5524

\section{AB0498 $\quad$ OPTIMISATION OF METHOTREXATE DOSE INDUCED SUCCESSFUL REDUCTION OF GLUCOCORTICOIDS WITHOUT IMPAIRED DISEASE CONTROL IN PATIENTS WITH RHEUMATOID ARTHRITIS}

S. Hirata ${ }^{1}$, T. Kondo ${ }^{1}$, K. Yukawa $^{1}$, T. Tokunaga ${ }^{1}$, T. Kuranobu ${ }^{1}$, K. Oi ${ }^{1}$, Y. Yoshida ${ }^{1}$, M. Funaki ${ }^{1,2}, K$. Oda ${ }^{1,3}$, T. Nojima ${ }^{1,4}$, E. Sugiyama ${ }^{1}{ }^{1}$ Department of Clinical Immunology and Rheumatology, Hiroshima University Hospital;

${ }^{2}$ Department of Rheumatology, JR Hiroshima Hospital; ${ }^{3}$ Hiroshima Clinic; ${ }^{4}$ Nojima Internal Medicine Clinic, Hiroshima, Japan

Background: Use of short-term glucocorticoids (GCs) along with methotrexate (MTX) have been recommended for newly onset patients with rheumatoid arthritis (RA) in EULAR recommendation 2016 However, it is not always easy to reduce or withdraw GCs due to patients' fear of relapsed pain or fatigue. As well, some patients are negative to increase MTX dose for fear of adverse events.

Objectives: To clarify whether GCs could be reduced without impaired disease control by optimising MTX dose in RA patients with stable medication in real-world clinical practice setting.

Methods: 70 patients with RA who regularly visit our outpatient clinic for $\geq 1$ year were enrolled. Clinical characters, disease activity, and medications at present and 1 year before were retrospectively collected. Therapeutic strategy was to increase MTX with reducing prednisolone (PSL) based on patient's consent. Initiating bDMARDs was allowed in case of uncontrollable disease. Wilcoxon test and chi-square test were used for statistics.

Results: Clinical characters (median [IQR]) were; age $62^{51,68} \mathrm{yrs}$; female $69 \%$; disease duration $6.8[3.4,13.7] \mathrm{yrs}$. Rate of MTX was elevated from $57 \%$ to $62 \%$, and dose (mean $\pm S D$ ) was increased from $9.8 \pm 3.2$ to $11.6 \pm 3.7 \mathrm{mg} / \mathrm{w}(\mathrm{p}<0.0001)$ for uses only, whereas PSL was suppressed from $56 \%$ to $26 \%$, and decreased from $2.0 \pm 3.1$ to $0.8 \pm 1.8 \mathrm{mg} / \mathrm{d}(\mathrm{p}=0.0004)$ for all patients. bDMARDs were used for 16 patients, and newly initiated for 2 patients. Although not significant, median CDAl, SDAl, and DAS28 were suppressed from 5.7 to $3.8,6.2$ to 3.9 , and 2.92 to 2.77 , and remission rate were increased from $24 \%$ to $39 \%, 27 \%$ to $41 \%$, and $36 \%$ to $41 \%$, respectively.

Abstract AB0498 - Table 1

\begin{tabular}{lccc}
\hline & Baseline & 1 Year & p-value \\
\hline$\%$ MTX & $57 \%$ & $67 \%$ & 0.2226 \\
MTX[mg/w] for users & $9.8 \pm 3.2$ & 11.6 & $<0.0001$ \\
& & \pm 3.7 & \\
\%PSL & $56 \%$ & $26 \%$ & 0.0003 \\
PSL[mg/d] for all & $2.0 \pm 3.1$ & $0.8 \pm 1.8$ & 0.0004 \\
patients & & & \\
CDAl remission & $24 \%$ & $39 \%$ & 0.0687 \\
SDAI remission & $27 \%$ & $41 \%$ & 0.075 \\
DAS28 remission & $36 \%$ & $41 \%$ & 0.4874 \\
\hline
\end{tabular}

Conclusions: GCs could be reduced or withdrawn without deterioration with appropriately increased MTX. Moreover, disease control rather showed improved tendency.

Disclosure of Interest: S. Hirata Grant/research support from: Eli Lilly, UCB, Consultant for: Bristol-Myers Squibb, UCB, Paid instructor for: AbbVie, Eisai, Tanabe-Mitsubishi, Speakers bureau: AbbVie, Astellas, Ayumi, Bristol-Myers Squibb, Chugai, Eisai, Eli Lilly, Jansen, Kissei, Pfizer, Sanofi, Takeda, Tanabe-Mitsubishi, UCB, T. Kondo: None declared, K. Yukawa: None declared, T. Tokunaga: None declared, T. Kuranobu: None declared, K. Oi: None declared, Y. Yoshida Speakers bureau: Bristol-Myers Squibb, Chugai, Sanofi, M. Funaki Speakers bureau: Chugai, K. Oda Speakers bureau: AsahiKasei, Kissei, Ono, Pfizer, Astellas, Jansen, Tanabe-Mitsubishi, T. Nojima Speakers bureau: Astellas, Ayumi, Bristol-Myers Squibb, Chugai, Sanofi, Tanabe-Mitsubishi, E. Sugiyama Grant/research support from: AbbVie, Astellas, Ayumi, Bristol-Myers Squibb, Chugai, Eisai, Eli Lilly, Kissei, Pfizer, Sanofi, Takeda, Tanabe-Mitsubishi, Speakers bureau: AbbVie, Astellas, Ayumi, Bristol-Myers Squibb, Chugai, Eli Lilly, Kissei, Pfizer, Sanofi, Tanabe-Mitsubishi, Actelion

DOI: 10.1136/annrheumdis-2018-eular.1382

\section{AB0499 EVALUATION OF THE EFFECTIVENESS OF METAXODINE IN THE HEPATIC TOXICITY DUE TO METHOTREXATE IN PATIENTS WITH RHEUMATOID ARTHRITIS}

S. Parisi, M.C. Ditto, M. Scarati, M. Priora, F. Enrico. Rheumatology Unit, AOU Città della Salute e della Scienza di Torino, Turin, Italy

Background: Methotrexate inhibits the metabolism of purines resulting in the accumulation of adenosine; it also inhibits the activation of $T$ cells and suppresses the expression of intercellular adhesion molecules for $\mathrm{T}$ cells. Side effects may show up during the treatment and among them there is hepatic toxicity, characterised by an increase of AST and ALT; such increase is usually asymptomatic but it may lead to a suspension of the treatment. Metadoxine (MTDX) is a drug which is used in order to treat both acute and chronic alcohol intoxication; it also prevents the inactivation of ATP from acetaldehyde and pyroglutamic acid. MTDX also showed to improve hepatic function markers and to decrease oxidative stress leading to a protective effect against radicals.

Objectives: The aim of this preliminary study was to evaluate the possible effect of MTDX on hepatic function in patients affected by RA in therapy with MTX Methods: The study involved the recruitment of patients affected by RA in treatment with MTX; a following random selection of a subgroup of patients who took MTDX (500 mg twice a day for 28 days, from the 5 th to the 8 th week of therapy with MTX) was performed. All the patients underwent a 12-week-follow up in which these parameters were evaluated: demographics, blood tests required for MTX in accordance with datasheets (especially AST and ALT), CRP, ESR, ACPA, numbers of swollen and tender joints, concomitant medications (NSAIDs and steroids) and the degree of disability (HAQ, table 1).

Results: 24 patients affected by RA ( 20 women), with an mean age of 51.3 years $( \pm 14.1)$ and mean MTX dose of $12.3 \pm 2.6$, were recruited. $70.3 \%$ took GC with a medium dosage $(3.72 \pm 2.71)$. Among these 24,13 patients were underwent MDTX $500 \mathrm{mg}$ twice a day from the 5 th to the 8th week. Patients treated with MTDX+MTX showed a significant decrease of hepatic markers (AST $\Delta-18.38$ $\mathrm{p}=0.004-$ ALT $\Delta-19.23 \mathrm{p}=0.004$ ) compare with patients with MTX only (AST $\Delta-4.27 \mathrm{p}=0.110-\mathrm{ALT} \Delta-6.09 \mathrm{p}=0.045$ ) after a 12-week-monitoring, with no statistically significant difference concerning disease activity (table 2). 
Abstract AB0499 - Table 1. Variables at baseline

\begin{tabular}{|c|c|c|c|}
\hline VARIABLES & MTX & MTX+MTDX & p-value \\
\hline Disease duration $(y y), m \pm S D$ & $4.91 \pm 1.70$ & $4.69 \pm 1.80$ & $n s$ \\
\hline$A S T(U / I), m \pm S D$ & $13.18 \pm 3.19$ & $18.46 \pm 7.15$ & $\mathrm{~ns}$ \\
\hline$A L T(U / I), m \pm S D$ & $17.09 \pm 6.35$ & $20.23 \pm 7.96$ & $\mathrm{~ns}$ \\
\hline VAS Pain, $m \pm S D$ & $23.64 \pm 19.76$ & $27.31 \pm 20.17$ & $\mathrm{~ns}$ \\
\hline$D A S 28, m \pm S D$ & $3.6 \pm 0.91$ & $3,72 \pm 0.87$ & $\mathrm{~ns}$ \\
\hline$H A Q, m \pm S D$ & $0.61 \pm 0.47$ & $0,68 \pm 0.37$ & $\mathrm{~ns}$ \\
\hline$M T X(m g), m \pm S D$ & $12.27 \pm 2.61$ & $12,69 \pm 2.59$ & $\mathrm{~ns}$ \\
\hline$G C(m g), m \pm S D$ & $3.41 \pm 2.80$ & $4,04 \pm 2.61$ & $\mathrm{~ns}$ \\
\hline
\end{tabular}

SD: standard deviation; ns: not significant; AST: aspartate amino transaminase; ALT:

alanine amino transaminase; MTX: methotrexate; MTDX: Metadoxine; GC: glucocorticoids

Abstract AB0499 - Table 2. Comparison of Hepatic Functions between two groups

\begin{tabular}{|c|c|c|c|c|c|c|}
\hline \multirow{2}{*}{ LIVER ENZYMES } & \multicolumn{3}{|c|}{ MTX } & \multicolumn{3}{c|}{ MTX+MTDX } \\
\cline { 2 - 7 } & $4 a \mathrm{wk}$ & $12 \mathrm{a} w \mathrm{k}$ & $\Delta$ & $4 \mathrm{a} w \mathrm{y}$ & $12 \mathrm{a} w \mathrm{y}$ & $\Delta$ \\
\hline$A S T(U / I), m \pm \mathrm{SD}$ & 39,09 & 34,82 & 4,27 & 42,46 & 24,08 & 18,38 \\
\hline$A L T(U / I), m \pm \mathrm{SD}$ & 46,82 & 40,73 & 6,09 & 43,92 & 24,69 & 19,23 \\
\hline
\end{tabular}

AST: aspartate amino transferase; ALT: alanine amino transferase; MTX: Methotrexate; MTDX: Metadoxine;

Conclusions: This study showed the possible effect of MTDX in increasing the tolerance to the MTX without affecting its effectiveness. Its role may indeed have useful implications in patients who start the therapy with MTX or in those who develop hepatic toxicity during the treatment.

\section{REFERENCE:}

[1] Fehér J, et al. The Beneficial Effect of Metadoxine in the Treatment of Fatty Liver Diseases. Reviews 2009;3(1):65-79.

Disclosure of Interest: None declared DOI: 10.1136/annrheumdis-2018-eular.6253

\section{AB0500 EFFICACY AND SAFETY OF TOFACITINIB (TOF) IN PATIENTS WITH RHEUMATOID ARTHRITISAT 52 WEEKS IN CLINICAL PRACTICE}

T. Aramaki ${ }^{1}$, K. Kojima ${ }^{1}$, S. Kurushima ${ }^{1}$, Y. Tsuji ${ }^{1}$, N. Kawachi ${ }^{1}$, N. Iwamoto ${ }^{2}$, K. Ichinose ${ }^{2}$, K. Terada ${ }^{1}$, K. Eguchi ${ }^{3}$, Y. Ueki ${ }^{1}$, A. Kawakami ${ }^{2}{ }^{1}$ Rheumatic Disease Center, Sasebo Chuo Hospital, Sasebo; ${ }^{2}$ Unit of Transklational Medicine, Department of Immunology and Rheumatology, Nagasaki Graduate School of Biomedical Sciences, Nagasaki; ${ }^{3}$ Sasebo Chuo Hospital, Sasebo, Japan

Objectives: There are few long-term experience of TOF for Rheumatoid arthritis (RA) patients in clinical use. We evaluated 52 and 104 weeks efficacy and safety of TOF in patients with rheumatoid arthritis in clinical practice.

Methods: We enrolled 77 RA patients started to treat with TOF between December 2013 and November 2016 in our hospital. All patients received $5 \mathrm{mg}$ of TOF twice daily. We evaluated clinical disease activity in composite measures (including Disease Activity Score 28(DAS28) -Erythrocyte (-ESR), - C-reactive protein (CRP), Simple Disease activity Index (SDAl) and Clinical Disease Activity Index (CDAI)) every three months and adverse events (AEs) during 52 and 104 weeks after treatment with TOF. We estimated the drug survival rate with Kaplan-Meier survival analysis.

Results: The mean age of patients was 66.3 years and the duration of disease was 15.4 years. Fifty-four $(74.0 \%)$ patients have positive rheumatoid factor (RF) and $59(78.7 \%)$ patients have positive anti-citrullinated potein antibodies (ACPA). Twenty-three patients were biologic-nave and fifty-four patients have ever been treated with biologic DMARDs of whose mean number is 2.6 prior to TOF. Regarding the last biologic DMARDs: 14 patients have treat with TNF inhibitor (Infliximab, etanercept, adalimumab, golimumab, certolizumab-pegol), 30 patients with tocilizumab and 10 patients with abatacept. Twenty-three $(29.9 \%)$ patients were treated with TOF without methotrextate (MTX). Disease activity at TOF start with measured with DAS28-ESR, DAS28-CRP, SDAI and CDAI were 5.15, 4.07, 23.3 and 21.5 respectively. The Drug survival rate at 24 weeks is $84.4 \%$ and that at 52 weeks is $73.8 \%$. Twenty-seven patients have been treated with tofacinib over 104 weeks. Twelve patients was stopped to treat within initial 24 week, including due to adeverse events $(n=5)$ and lack of efficacy $(n=7)$. Analysed by last observation continued forward (LOCF) method, the mean of DAS-ESR decreased to 3.52 at 24 week and 3.59 at 52 week. CDAl decreased from 21.5 to 7.94 at 24 week and
7.93 at 52 week. Sixteen (20.8\%) patients were remission as which defined less than 2.6 in DAS-ESR and $13(16.9 \%)$ were low disease activity (LDA) as which defined less than 3.2 in DAS-ESR. In 23 patients without MTX, $6(26.0 \%)$ and 5 $(21.7 \%)$ have achieved remission and LDA at $52 \mathrm{~W}$ respectively. The adverse events due to which the patients had to be stopped to treat with TOF occurred in $20.6 \%$ of patients in initial 52 weeks. Infection was the most frequent AE to TOF, especially herpes zoster (7 patients). Six patients experienced herpes zoster within 6 month after treat with TOF. All of them could restart to treatment of TOF. Two solid tumours were discovered within 6 months after treatment, both of them had be removed by surgery and has not recurred.

Conclusions: Our study shows that TOF can be much effective clinically and have longer-lasting effectiveness. In our patients, herpes zoster was almost as frequent as reported in the phase III trials in Japan and we could retreat almost of them with TOF.

Disclosure of Interest: None declared

DOI: 10.1136/annrheumdis-2018-eular.3863

\section{AB0501 ADVERSE EVENTS WITH TREATMENT REGIMENS OF RHEUMATOID ARTHRITIS (RA) REPORTED BY PATIENTS APPLYING SMART SYSTEM OF DISEASE MANAGEMENT (SSDM) MOBILES TOOLS: A COHORT STUDY OF RA PATIENTS IN CHINA}

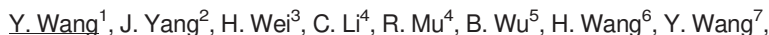
X. Wang ${ }^{8}$, H. Guo ${ }^{9}$, J. Xue ${ }^{10}$, J. Ru ${ }^{11}$, X. Xin ${ }^{12}$, F. Zhang ${ }^{13}$, L. Kang ${ }^{1}$, H. Liu ${ }^{2}$, J. YuHua ${ }^{14}$, Y. Liu ${ }^{14}$, F. Xiao ${ }^{15}$, J. Huang ${ }^{16} .{ }^{1}$ The First Affiliated Hospital of BaoTou Medical College, Bao Tou; ${ }^{2}$ Central Hospital of Mian Yang, Sichuan, MianYang; ${ }^{3}$ Northern Jiangsu People's Hospital, Yang Zhou; ${ }^{4}$ People's Hospital, Beijing University Medical School, Beijing, ${ }^{5}$ Chongqing Traditional Chinese Medicine Hospital, Chongqing; ${ }^{6}$ JiaXing First Hospital, Zhejiang, JiaXing; ${ }^{7}$ Southwest Hospital, Chongqing; ${ }^{8}$ Anyang District Hospital, Puyang; ${ }^{9}$ The Second Hospital of Hebei Medical University, Shijiazhuang; ${ }^{10}$ The Second Affiliate Hospital of Zhejiang University School of Medicine, Hangzhou; ${ }^{11}$ The 264th Hospital of the PLA, Taiyuan; ${ }^{12}$ NingBo First Hospital, Zhejiang, Ningbo; ${ }^{13} \mathrm{Hebei}$ General Hospital, Shijiazhuang; ${ }^{14}$ Shanghai Gothic Internet Technology Co., Ltd; ${ }^{15}$ Suzhou antiinflammatory research institue, Shanghai; ${ }^{16}$ The sixth Affiliated Hospital of Sun Yat-sen University, Guang Zhou, China

Background: Hepatic, hematologic and other adverse events $(\mathrm{AE})$ during treatment in RA patients are unavoidable. And monitoring AE in long-term treatment is quite necessary as a part of chronic disease management. SSDM is a smart mobile tool to help patients upload their therapeutic regimens, lab test records and report AEs. Our previous study showed that patients in China can master the application of SSDM after training.

Objectives: To determine and compare the incidence of adverse events during treatment of RA with different therapeutic regimens, focusing on mono and combination therapy.

Methods: The SSDM includes interfaces of both physicians' and patients' application. Patients were educated to enter the data of lab test records and treatment regiments once a month, all data can be synchronised automatically to the authorised physicians' mobile tool, which formed a large patient reported database. The rheumatologists can also adjust treatment regiments base on patients' profile.

Results: From Aug 2014 to Jan 2018, a total of 7048 RA patients from 480 centres in China were entered in the cohort study. These patients contributed more than 12600 patient-years (PY) of total followup. The mean age was 48.98 \pm 16.08 ( 18 to 99 ) years and the median disease duration was 23.27 months. The treatment regimens include mono or combination of leflunomide(LEF), MTX, hydroxychologine (HCQ), sulfasalazine(SSZ), glucocorticoid(GC), biologic DMARD, Tripterygium wilfordii, meloxicam, celecoxib, iguratimod, etc. In this database the five most common treatment regimens is LEF monotherapy (3801 PY), MTX monotherapy (1321 PY), LEF +MTX (1086 PY), HCQ monotherapy (715 PY), LEF +HCQ (576 PY). The incidence rate of hepatic events was lower for LEF monotherapy (35 events/1000 PY) than MTX (52 events/1000 PY) and LEF + MTX combination therapy (115 events/1000 PY) $(p<0.01)$. The incidence rate of leukopenia was lower for LEF monotherapy (42 events/1000 PY) and MTX monotherapy (39 events/1000 PY) than LEF +MTX combination therapy (84 events/ $1000 \mathrm{PY})(\mathrm{p}<0.01)$.

Conclusions: The findings show that mono or combination of csDMARDs are the most commonly used drugs in Chinese RA patients. And AEs may be well described in this patient report database because of the large sample sizes and empowering patient themselves. RA patients can get better safety in the longterm treatment via SSDM.

Disclosure of Interest: None declared

DOI: 10.1136/annrheumdis-2018-eular.6333 